\title{
ANATOMÍA COMPARADA DE LA LÁMINA FOLIAR DEL GÉNERO DISTICHLIS (POACEAE)
}

\author{
María Martina López Soto, Stephen D. Koch, \\ María Flores-Cruz y E. Mark Engleman
}

Colegio de Postgraduados, Instituto de Recursos Naturales, Programa de Botánica, 56230 Montecillo, Estado de México, México.sdkoch@colpos.mx

\section{RESUMEN}

Se hizo un estudio anatómico-sistemático de seis especies y 11 variedades del género Distichlis Raf. Los taxa incluidos fueron: D. australis (Speg.) Villamil; D. humilis Phil.; D. palmeri (Vasey) Fassett ex I. M. Johnst.; D. scoparia (Nees ex Kunth) Arechav. var. erinacea (Beetle) Nicora, y var. scoparia; y D. spicata (L.) Greene, esta última representada por las siguientes ocho variedades: var. borealis (J. Presl) Beetle, var. divaricata Beetle, var. mendocina (Phil.) Hack., var. mexicana Beetle, var. nana Beetle, var. spicata, var. stolonifera Beetle y var. stricta (Torr.) Scribn. Se incluyeron dos taxa cuya información anatómica fue extraída de la literatura: Distichlis laxiflora Hack. y Distichlis spicata var. distichophylla (Michx.) Beetle. Se encontraron 14 caracteres anatómicos considerados de valor taxonómico, siete en corte transversal y siete en vista superficial de la epidermis abaxial. Distichlis australis y D. palmeri difieren claramente del resto de las especies. Distichlis humilis, D. scoparia y D. spicata forman un grupo anatómicamente homogéneo pero con características que las hacen distintivas a cada una. Para Distichlis spicata, no se encontró ningún carácter o combinación de caracteres anatómicos con los cuales pueda separarse en distintos grupos. Por esta razón, las especies, subespecies y/o variedades propuestas por algunos autores para esta entidad se consideran sinónimos de D. spicata.

Palabras clave: anatomía, Distichlis, lámina foliar, Poaceae, taxonomía.

\section{ABSTRACT}

Six species and 11 varieties of the genus Distichlis Raf. were compared anatomically. The taxa studied were: Distichlis australis (Speg.) Villamil; D. humilis Phil.; D. palmeri 
(Vasey) Fassett ex I.M. Johnst.; D. scoparia (Nees ex Kunth) Arechav. var. erinacea (Beetle) Nicora and var. scoparia; and D. spicata (L.) Greene, represented by the following eight varieties: var. borealis (J. Presl) Beetle, var. divaricata Beetle, var. mendocina (J. Presl) Hack., var. mexicana Beetle, var. nana Beetle, var. spicata, var. stolonifera Beetle and var. stricta (Torr.) Scribn. Also included were two taxa for which the anatomical information was taken from the literature: Distichlis laxiflora Hack. and Distichlis spicata var. distichophylla (Michx.) Beetle. Fourteen anatomical characters of taxonomic value were found, seven in cross section and seven in the epidermis as seen in surface view. Distichlis australis and D. palmeri were found to differ from all of the other species. Distichlis humilis, D. scoparia and D. spicata form an anatomically homogeneous group, but with characteristics that distinguish each of them. Within Distichlis spicata, no anatomical character or combination of characters was found that would justify the recognition of infraspecific taxa and/or segregate species. For this reason, these taxa are considered to be synonyms of D. spicata.

Key words: anatomy, Distichlis, leaf blade, Poaceae, taxonomy.

\section{INTRODUCCIÓN}

El género Distichlis fue propuesto por Rafinesque en 1819 para un pequeño grupo de plantas herbáceas, dioicas, perennes y rizomatosas, con espiguillas multifloras y hojas conspicuamente dísticas que habitan suelos húmedos y salinos. Pertenece a la familia Poaceae, subfamilia Chloridoideae, tribu Cynodonteae y subtribu Monanthochloineae (Peterson et al., 2001). Se distribuye en las costas y el interior del continente americano y una de sus especies se registra de Australia (Beetle, 1943; Nicora y Rúgolo de Agrasar, 1987; Beetle et al., 1991; Watson y Dallwitz, 1992; Zuloaga et al., 1994). De acuerdo con Beetle (1943), Burkart et al. (1969), Nicora (1978), McVaugh (1983), Peterson et al. (2001) y Rzedowski (2001), el género incluye entre tres y siete especies y hasta doce subespecies y variedades, lo que indica que existen diferencias sustanciales entre varios autores respecto a la clasificación a nivel infragenérico.

Debido a que todas las especies son muy semejantes en su morfología, pero a un nivel más fino existe polimorfismo, se originaron dos tendencias en la clasificación de Distichlis: una, en la que se reconocen pocas especies, cada una con mucha variación, y otra, en la que se consideran muchas entidades específicas y subespecíficas difíciles de distinguir. Hasta el presente, las características empleadas para definir los taxa en el género han sido casi exclusivamente morfológicas. Con el fin 
de intentar resolver las dificultades anteriormente mencionadas, se propuso revisar el grupo utilizando una nueva fuente de información como lo es la anatomía vegetal comparada. Así, se estudiaron los rasgos de la epidermis abaxial y la estructura interna de la lámina foliar de las especies y variedades propuestas.

Existen algunos estudios anatómicos realizados para el género Distichlis, pero aunque contribuyen al conocimiento del grupo, incluyen pocas especies y su orientación no es con el fin de resolver los problemas taxonómicos dentro del mismo. Holm (1891 y 1901-1902) describe la estructura anatómica de una serie de individuos masculinos y femeninos de varias especies de Distichlis en diferentes localidades de los Estados Unidos de América. Metcalfe (1960) describe la anatomía de la lámina foliar de Distichlis spicata var. distichophylla como un ejemplo del género en el contexto de un estudio comparativo de toda la familia Poaceae.

Villamil (1969) estudia las dos especies sudamericanas de Monanthochloë Engelm., un género muy relacionado con Distichlis (Watson y Dallwitz, 1992), y transfiere a Monanthochloë australis a Distichlis.

Nicora (1978) estudia la anatomía de D. laxiflora y D. scoparia y concluye, con base en su estudio, que la primera debe ser reconocida como una especie diferente de la segunda.

Watson y Dallwitz (1992) presentan una diagnosis anatómica del género Distichlis, pero esta es muy general y no contribuye a la resolución de los problemas taxonómicos dentro del género.

\section{MATERIALES Y MÉTODOS}

Para los propósitos de este trabajo se examinaron seis especies y 11 variedades del género Distichlis (Cuadro 1).

El material utilizado proviene de ejemplares de los siguientes herbarios (abreviaturas según Holmgren et al., 1990): CAS, CHAPA, DS, MEXU, UC, UNC y US.

Se estudiaron 200 ejemplares (ver Apéndice). De Distichlis laxiflora, D. spicata var. distichophylla y var. andina Beetle, no se encontraron especímenes en los herbarios a los que se solicitaron los préstamos; sin embargo para las dos primeras se tomaron datos anatómicos de la literatura.

Los cortes transversales y las epidermis abaxiales se obtuvieron manualmente utilizando una modificación del método de Metcalfe (1960). Después de establecer que no se notaban diferencias entre las hojas de diferentes nudos a lo largo del tallo, 
Cuadro 1. Especies y variedades examinadas del género Distichlis.

\begin{tabular}{lll}
\hline \multicolumn{1}{c}{ Norteamérica } & \multicolumn{1}{c}{ Sudamérica } & \multicolumn{1}{c}{ Australia } \\
\hline D. palmeri (Vasey) Fassett & D. australis (Speg.) Villamil & D. spicata var. \\
ex I.M. Johnst. & D. humilis Phil. & distichophylla \\
D. spicata (L.) Greene & D. laxiflora Hack. & \\
var. borealis (J. Presl) & D. scoparia (Nees ex Kunth) \\
Beetle & Arechav. \\
var. divaricata Beetle & var. erinacea (Beetle) Nicora \\
var. mexicana Beetle & var. scoparia \\
var. nana Beetle & D. spicata \\
var. spicata & var. mendocina (Phil.) Hack. \\
var. stolonifera Beetle & var. stricta (Torr.) Scribn. \\
var. stricta (Torr.) Scribn. & \\
\hline
\end{tabular}

se tomaron al azar láminas foliares sanas y maduras. El tercio central de cada una de ellas se hidrató hirviéndolo en agua durante aproximadamente seis minutos. Posteriormente se colocó el fragmento de hoja sobre un vidrio bajo un microscopio estereoscópico y con una navaja de afeitar se realizaron los cortes transversales. Para observar la epidermis abaxial aislada, se rasparon y eliminaron la epidermis adaxial, el mesófilo y el tejido vascular. Tanto los cortes transversales como las epidermis abaxiales aisladas se introdujeron en hipoclorito de sodio comercial a 5\% y se dejaron de cinco a diez minutos para aclararlos. Posteriormente, se lavaron y montaron sin tinción en gelatina glicerinada (Curtis, 1986).

En Distichlis palmeri adicionalmente se hicieron cortes longitudinales de las hojas, debido a que las papilas cubren por completo los micropelos, dificultando su observación en vista superficial. Para obtener las muestras de D. palmeri, se colocó un fragmento de la porción media de una lámina foliar (previamente hidratado) en ácido fluorhídrico a $8 \%$ durante 24 horas para remover el sílice, procesando el material de acuerdo con la metodología propuesta por Johansen (1940), modificada por E. M. Engleman (datos no publicados). Ésta consiste en la deshidratación gradual de la muestra en una serie de alcoholes y la infiltración con xileno, en un cambiador automático de tejidos. Se le incluyó en parafina y se hicieron cortes de 10 micrómetros de espesor en un micrótomo. Se tiñeron con safranina-verde fijo y se montaron en bálsamo de Canadá.

En un microscopio compuesto a 100, 400 y 1000x, se hicieron las observaciones de la estructura anatómica en las preparaciones de los taxa estudiados. 
Se realizaron comparaciones de la estructura de los ejemplares femeninos con la de los masculinos, con la finalidad de determinar la correlación de los sexos con la anatomía. El sexo de cada planta estudiada está anotado en el Apéndice y también en las leyendas de las figuras.

Las mediciones realizadas son relativas; se expresan como proporciones entre dos células o partes de una misma célula, como la longitud de la base del aguijón marginal con respecto a la longitud de un estoma de la misma lámina foliar y la longitud del ápice con respecto a la base del mismo aguijón marginal.

La terminología utilizada en el texto, los cuadros y leyendas de las figuras fue tomada de Ellis $(1976,1979)$. Los dibujos se realizaron con ayuda de una cámara lúcida.

Para la especie Distichlis spicata, además de estudiar materiales procedentes de diversas localidades del continente americano, se hicieron observaciones anatómicas de varias láminas foliares de tallos de un mismo rizoma. Los rizomas se recolectaron en el ex-lago de Texcoco, en diferentes condiciones ambientales. Lo anterior se hizo con la finalidad de determinar la variación de caracteres dentro de un mismo individuo.

\section{RESULTADOS Y DISCUSIÓN}

Las observaciones anatómicas realizadas en ejemplares masculinos y femeninos no muestran diferencias significativas entre los dos sexos de las plantas a este respecto examinadas.

Se identificaron 14 caracteres anatómicos con valor taxonómico para diferenciar las especies estudiadas (Cuadros 2 y 3 ).

Distichlis australis se separa anatómicamente de los miembros restantes del género, por poseer únicamente haces vasculares considerados como secundarios debido a que no presentan vasos metaxilemáticos notables (Fig. 1A, B; Cuadro 2). Esta observación concuerda con la registrada por Villamil (1969) y Nicora (1978) para el género Monanthochloë. En Distichlis australis, las células buliformes se encuentran en la base de cada surco adaxial y además forman parte de casi toda la epidermis, excepción hecha de las costillas marginales (Fig. 1B; Cuadro 2). Frecuentemente las células largas intercostales abaxiales poseen papilas de gran longitud que se llegan a traslapar unas con otras y en ocasiones cubren parcialmente a los micropelos (Fig. 1D). Los caracteres anatómicos observados en este estudio apoyan la inclusión de este taxon en el género Monanthochloë. Lo anterior concuerda con la opinión de Watson y Dallwitz (1992). 
Cuadro 2. Caracteres diferenciales en corte transversal de la lámina foliar de las especies del género Distichlis.

\begin{tabular}{|c|c|c|c|c|c|}
\hline & $\begin{array}{c}\text { Distichlis } \\
\text { australis }\end{array}$ & $\begin{array}{c}\text { Distichlis } \\
\text { palmeri }\end{array}$ & $\begin{array}{l}\text { Distchlis } \\
\text { humilis }\end{array}$ & $\begin{array}{l}\text { Distchlis } \\
\text { scoparia }\end{array}$ & $\begin{array}{l}\text { Distchlis } \\
\text { spicata }\end{array}$ \\
\hline Número de costillas & $7-9$ & $12-25$ & $11-18$ & $7-11$ & $12-28$ \\
\hline \multicolumn{6}{|l|}{$\begin{array}{l}\text { Número de haces } \\
\text { vasculares }\end{array}$} \\
\hline Primarios & 0 & $5-10$ & $3-4$ & $3-5$ & $4-9$ \\
\hline Secundarios & $7-9$ & $9-13$ & $8-14$ & $2-6$ & $7-15$ \\
\hline $\begin{array}{l}\text { Extensión de la vaina } \\
\text { externa de los haces } \\
\text { vasculares primarios } \\
\text { y secundarios }\end{array}$ & ausente & $\begin{array}{l}\text { presente en } \\
\text { todos los haces } \\
\text { primarios y } \\
\text { secundarios }\end{array}$ & ausente & $\begin{array}{l}\text { ausente } \\
\text { u ocasio- } \\
\text { nalmente } \\
\text { presente en } \\
\text { unos haces }\end{array}$ & $\begin{array}{l}\text { ausente } \mathrm{u} \\
\text { ocasionalmen- } \\
\text { te presente en } \\
\text { unos haces }\end{array}$ \\
\hline $\begin{array}{l}\text { Cuello de la célula } \\
\text { basal del micropelo }\end{array}$ & presente & ausente & presente & presente & presente \\
\hline $\begin{array}{l}\text { Distribución de } \\
\text { células buliformes }\end{array}$ & $\begin{array}{l}\text { en toda la } \\
\text { epidermis } \\
\text { adaxial }\end{array}$ & surcos adaxiales & $\begin{array}{l}\text { surcos } \\
\text { adaxiales }\end{array}$ & $\begin{array}{l}\text { surcos } \\
\text { adaxiales }\end{array}$ & $\begin{array}{l}\text { surcos } \\
\text { adaxiales }\end{array}$ \\
\hline $\begin{array}{l}\text { Distribución del } \\
\text { esclerénquima } \\
\text { marginal }\end{array}$ & $\begin{array}{l}\text { paquete } \\
\text { aislado }\end{array}$ & $\begin{array}{l}\text { fusionado al } \\
\text { esclerénqui- } \\
\text { ma adaxial y } \\
\text { abaxial adya- } \\
\text { cente }\end{array}$ & $\begin{array}{l}\text { paquete } \\
\text { aislado }\end{array}$ & $\begin{array}{l}\text { paquete } \\
\text { aislado }\end{array}$ & $\begin{array}{l}\text { paquete } \\
\text { aislado }\end{array}$ \\
\hline
\end{tabular}

Las plantas pertenecientes a Distichlis palmeri tienen características diferenciales que hacen a esta especie única dentro del género. En el mencionado taxon se observó que la extensión de la vaina externa (Fig. 2A, B; Cuadro 2) siempre está presente en los haces vasculares primarios y secundarios, en contraste con las especies restantes en las cuales esta estructura está ausente u ocasionalmente presente. El esclerénquima está bien desarrollado en el margen y se encuentra fusionado al esclerénquima adaxial y abaxial de la costilla marginal (Fig. 2B). En ocasiones, éste se fusiona además al esclerénquima abaxial de la costilla adyacente a la marginal. Otras características se observan en vista superficial de la epidermis abaxial. Una de ellas es la aparente ausencia de células de corcho (Fig. 2C; Cuadro 3); otra consiste en los cuerpos de sílice (Fig. 2C; Cuadro 3), que por lo común son angulares y muestran una variedad de formas. Lo anterior contrasta con 
las figuras generalmente de contorno redondeado que se encuentran en las demás especies estudiadas (Cuadro 3). Las papilas de las células intercostales asociadas a los micropelos suelen ser mucho más largas que las de las células intercostales restantes y frecuentemente forman grupos muy conspicuos que cubren totalmente a estas estructuras (Fig. 2C). El micropelo (Fig. 2A y D; Cuadro 2) constituye otro carácter de valor taxonómico, debido a que la célula basal carece de un cuello sobresaliente de la epidermis, como en los otros representantes de Distichlis. Los aguijones marginales (Cuadro 3) faltan en D. palmeri, mientras que siempre se observan en las especies restantes.

Distichlis humilis (Fig. 3), D. scoparia (Fig. 4) y D. spicata (Figs. 5, 6, 7 y 8) poseen caracteres anatómicos similares (Cuadros 2 y 3), pero muestran rasgos distintivos en el número de costillas y de haces vasculares secundarios (Cuadro 2), así como en la morfología de los aguijones marginales (Cuadro 3). Distichlis scoparia presenta el menor número de costillas (7 a 11) y de haces vasculares secundarios (2 a 6), en contraste con $D$. humilis, en la cual se observaron 11 a 18 y 8 a 14 respectivamente; en D. spicata se cuantificaron 12 a 28 y 7 a 15 . La longitud del ápice de los aguijones marginales, comparada con la de su base y con el tamaño del estoma (Cuadro 3), también sirven para distinguir a estas tres especies. En D. humilis, el ápice de los aguijones marginales es generalmente más de cuatro veces más largo que la base siendo ésta más corta que un estoma (Fig. 3D; Cuadro 3), mientras que en D. scoparia (Fig. 4C) las bases de los aguijones siempre son del mismo largo o un poco mayores que un estoma y en $D$. spicata (Figs. 5C, 6C, 7C y 8C; Cuadro 3) se presentan con una base generalmente más de dos veces más larga que un estoma (Fig. 7C). La longitud del ápice de los aguijones en D. scoparia y D. spicata es generalmente menos de tres veces mayor que la de la base (Cuadro 3).

En sus rasgos anatómicos los ejemplares correspondientes a $D$. scoparia var. scoparia y D. scoparia var. erinacea son similares y no se observó ningún carácter de valor sistemático con el cual se pudiera hacer una distinción entre ellas.

Distichlis laxiflora fue incluida por Beetle (1955) como una variedad de Distichlis scoparia. Sin embargo, Nicora (1978) considera que esta planta debe ser reconocida como una especie distinta, ya que tiene suficientes características anatómicas que la separan de D. scoparia. Según esta autora, en D. laxiflora las hojas son convolutas, la costilla formada por el haz medio es regularmente plana o redondeada en la cara adaxial y mayor que las que forman los haces restantes, y todos los haces están unidos por esclerénquima a ambas epidermis. En contraste, D. scoparia, tiene hojas conduplicadas, el haz medio es menor que los restantes haces vasculares primarios, la costilla que lo contiene es de forma triangular y los 
Cuadro 3. Caracteres diferenciales en vista superficial de la epidermis abaxial de la lámina foliar de las especies del género Distichlis.

\begin{tabular}{|c|c|c|c|c|c|}
\hline & $\begin{array}{l}\text { Distichlis } \\
\text { australis }\end{array}$ & $\begin{array}{c}\text { Distichlis } \\
\text { palmeri }\end{array}$ & $\begin{array}{c}\text { Distichlis } \\
\text { humilis }\end{array}$ & $\begin{array}{l}\text { Distichlis } \\
\text { scoparia }\end{array}$ & $\begin{array}{c}\text { Distichlis } \\
\text { spicata }\end{array}$ \\
\hline $\begin{array}{l}\text { Longitud de las } \\
\text { papilas intercos- } \\
\text { tales asociadas a } \\
\text { micropelos en com- } \\
\text { paración con la de } \\
\text { las papilas de otras } \\
\text { células intercostales }\end{array}$ & igual & $\begin{array}{l}\text { mucho } \\
\text { mayor }\end{array}$ & igual & mayor & igual \\
\hline $\begin{array}{l}\text { Cobertura de mi- } \\
\text { cropelos por papilas }\end{array}$ & nula o parcial & completa & nula o parcial & nula o parcial & nula o parcial \\
\hline Células de corcho & presentes & ausentes & presentes & presentes & presentes \\
\hline $\begin{array}{l}\text { Forma de los cuer- } \\
\text { pos de sílice }\end{array}$ & redondeados & angulares & redondeados & redondeados & redondeados \\
\hline $\begin{array}{l}\text { Aguijones margi- } \\
\text { nales }\end{array}$ & presentes & ausentes & presentes & presentes & presentes \\
\hline $\begin{array}{l}\text { Longitud de la base } \\
\text { de los aguijones } \\
\text { marginales compa- } \\
\text { rada con la longitud } \\
\text { de las células de } \\
\text { guarda }\end{array}$ & $\begin{array}{l}1.5-2 \text { veces } \\
\text { más largos }\end{array}$ & ausentes & $\begin{array}{l}\leq \text { a células } \\
\text { de guarda }\end{array}$ & $\begin{array}{l}\text { = a células } \\
\text { de guarda }\end{array}$ & $\begin{array}{l}\geq 2 \text { veces } \\
\text { más largos }\end{array}$ \\
\hline $\begin{array}{l}\text { Longitud del ápice } \\
\text { de los aguijones } \\
\text { marginales con } \\
\text { respecto a su base }\end{array}$ & $\begin{array}{l}1-3 \text { veces } \\
\text { más largo }\end{array}$ & ausentes & $\begin{array}{l}\geq 4 \text { veces } \\
\text { más largo }\end{array}$ & $\begin{array}{l}<3 \text { veces } \\
\text { más largo }\end{array}$ & $\begin{array}{l}<3 \text { veces } \\
\text { más largo }\end{array}$ \\
\hline
\end{tabular}

haces vasculares menores son libres o están unidos por esclerénquima únicamente a la epidermis abaxial.

En D. scoparia la forma de la cara adaxial de la costilla de la vena media es redondeada a ligeramente triangular y los haces vasculares de la vena media son de tamaño similar al de los haces vasculares primarios restantes (Figs. 4A y 4B). Lo anterior no concuerda con las observaciones realizadas por Nicora (1978) para esta especie. El esclerénquima adaxial en $D$. scoparia se observa libre en los haces vasculares secundarios y terciarios y ocasionalmente unido a los haces vasculares primarios como menciona Nicora (1978). 
Distichis laxiflora, como se describió anteriormente, fue reconocida por Beetle (1955) como una variedad de D. scoparia, y como una entidad distinta por Nicora (1978). No obstante, se reconoce que las características que describe la última autora para distinguir a tal especie de $D$. scoparia corresponden, según el presente estudio, a la amplia variación anatómica registrada para $D$. spicata, y no a los rasgos de $D$. scoparia. Por lo anterior, se debe considerar la posibilidad de que estas plantas sean miembros de D. spicata (Figs. 5A, 6A, 7A y 8A).

Con respecto a Distichlis spicata var. distichophylla, los datos anatómicos presentados por Metcalfe (1960) sugieren que esta planta no es sino una variante de D. spicata. El mencionado autor toma a este taxon como representante del género, proporcionando las siguientes características: células cortas sobre y entre las venas; cuerpos de sílice sobre las venas de formas cuboides, redondos o algunas veces casi en forma de silla de montar; micropelos presentes y aparentemente esféricos y "unicelulares" (sic); estomas con células subsidiarias triangulares o en forma de domo; haces vasculares generalmente pequeños con contorno interno moderadamente angular; mesófilo con clorénquima radiado y vainas de los haces vasculares dobles y "simples" (sic). Con base en lo anterior, se infiere que la estructura anatómica de $D$. spicata var. distichophylla es similar a la del material observado de D. spicata en el continente americano.

Se encontraron diversas características microscópicas con valor para separar las especies del género Distichlis. Sin embargo, las variedades de D. spicata propuestas o aceptadas por Beetle $(1943,1945,1955)$ y Beetle et al. (1991) no se reconocen debido a la ausencia de diferencias anatómicas consistentes (Figs. 5, 6, 7 y 8). Para sustentar esta idea, se observó en diferentes plantas de $D$. spicata una variabilidad extrema en la estructura interna de diversas láminas foliares de varios tallos de un mismo rizoma. Por tal razón, se concluye que las características distintivas de las variedades reconocidas, sólo representan respuestas plásticas de un solo genotipo a diferentes condiciones ambientales.

En general los rasgos anatómicos en las variedades propuestas para la especie Distichlis spicata son similares pero muestran límites de variabilidad muy amplios sin encontrar discontinuidades, como también fue observado por Holm (1891, 1901-1902). Lo anterior no permite la posibilidad de dar un carácter o caracteres especiales con los cuales se puedan separar los grupos establecidos como especies segregadas, subespecies o variedades que han propuesto algunos autores (Beetle, 1943, 1945, 1955; Beetle et al., 1991; Fassett, 1925; Hitchcock, 1935, 1951; Peterson et al., 2001; Rydberg, 1909). 


\section{CONCLUSIONES}

La herramienta utilizada en este estudio dio buenos resultados para distinguir las especies del género Distichlis. Se encontraron 14 caracteres anatómicos considerados de valor taxonómico, siete en corte transversal y siete en vista superficial de la epidermis abaxial.

De acuerdo con los resultados obtenidos, $D$. australis y $D$. palmeri presentan caracteres anatómicos propios que los hacen únicos y diferentes del resto de las especies, por lo que es probable que estos taxa estén mal ubicados en el género Distichlis. Distichlis australis está más relacionado con el género Monanthochloë por poseer haces vasculares secundarios, y no primarios como en el resto de los taxa de Distichlis. Las afinidades de D. palmeri no son claras.

Distichlis humilis, D. scoparia y D. spicata comprenden un grupo anatómicamente homogéneo, pero tienen caracteres que permiten diferenciar a cada especie.

La información encontrada no apoya la subdivisión de Distichlis spicata en grupos taxonómicos, como algunos estudiosos han pretendido al proponer la existencia de varias especies, subespecies y/o variedades. Esta investigación respalda el concepto de la gran mayoría de agrostólogos y autores de tratados florísticos, quienes consideran que tales variedades no están suficientemente definidas para merecer reconocimiento como taxa distintos.

\section{AGRADECIMIENTOS}

Nuestro especial agradecimiento al Consejo Nacional de Ciencia y Tecnología, por el apoyo económico brindado a la primera autora (beca 91503), así como a los curadores y personal de los herbarios, de los que se solicitaron los ejemplares empleados en este trabajo. El curador del herbario CANB, tuvo la amabilidad de enviarnos el material fotográfico correspondiente a D. spicata var. distichophylla (Michx.) Beetle. Finalmente, agradecemos de manera especial al editor por haber mejorado notablemente el texto.

\section{LITERATURA CITADA}

Beetle, A. A. 1943. The North American variations of Distichlis spicata. Bull. Torrey Bot. Club 70: 638-650. 
Beetle, A. A. 1945. Distichlis spicata in Australia. Rhodora 47: 148.

Beetle, A. A. 1955. The grass genus Distichlis. Rev. Argentina Agron. 22: 86-94.

Beetle, A. A., A. N. Córdova y R. A. Flores. 1991. Gramíneas de Sonora, México. Comisión Técnico Consultiva para la Determinación Regional de los Coeficientes de Agostadero, Secretaría de Agricultura y Recursos Hidráulicos. México, D.F. 174 pp.

Burkart, A., A. C. José, A. O. Katsuo, R. A. Palacios, Z. E. Rúgolo, E. S. García, M. Toursarkissian y N. S. Troncoso. 1969. Flora Ilustrada de Entre Ríos (Argentina). Vol. II. Gramíneas. Instituto Nacional de Tecnología Agropecuaria. Buenos Aires, Argentina. 537 pp.

Curtis, J. 1986. Microtecnia vegetal. Editorial Trillas. México, D.F. 106 pp.

Ellis, R. P. 1976. A procedure for standardizing leaf anatomy in the Poaceae. I. The leafblade as viewed in transverse section. Bothalia 12: 65-109.

Ellis, R. P. 1979. A procedure for standardizing leaf anatomy in the Poaceae. II. The epidermis as seen in surface view. Bothalia 12: 641-671.

Fassett, N. C. 1925. Notes on Distichlis. Rhodora 27: 67-72.

Hitchcock, A. S. 1935. Manual of the grasses of the United States. United States Department of Agriculture Miscellaneous Publication 200. 1040 pp.

Hitchcock, A. S. 1951. Manual of the grasses of the United States. 2a. ed. revised by A. Chase. United States Department of Agriculture Miscellaneous Publication 200. $1051 \mathrm{pp}$.

Holm, T. 1891. A study of some anatomical characters of North American Gramineae. III. Distichlis and Pleuropogon. Bot. Gaz. 16: 277-281.

Holm, T. 1901-1902. Some new anatomical characters for certain Gramineae. Beih. Bot. Centralbl. 11: 101-133.

Holmgren, P. K., N. H. Holmgren y L. C. Barnett. 1990. Index herbariorum I: The herbaria of the world. 8th ed. New York Botanical Garden. Nueva York. 693 pp.

Johansen, D. A. 1940. Plant microtechnique. McGraw Hill. Nueva York. 523 pp.

McVaugh, R. 1983. Flora Novo-Galiciana. Vol. 14. Gramineae. The University of Michigan Press. Ann Arbor. 436 pp.

Metcalfe, C. R. 1960. Anatomy of monocotyledons. I. Gramineae. Oxford University Press. Oxford. $731 \mathrm{pp}$.

Nicora, E. G. 1978. Gramineae. In: Correa, M. N. Flora Patagónica 8(3): 1-563.

Nicora, E. G. y E. Z. Rúgolo de Agrasar. 1987. Los géneros de gramíneas de América Austral. Editorial Hemisferio Sur. Buenos Aires, Argentina. 611 pp.

Peterson, P. M., R. J. Soreng, G. Davidse, T. S. Filgueiras, F. O. Zuloaga y E. Judziewicz. 2001. Catalogue of New World grasses (Poaceae): II. Subfamily Chloridoideae. Contrib. U. S. Natl. Herb. 41: 1-255.

Rafinesque, C. S. 1819. Prodrome des nouveaux genres de plantes observés en 1817 et 1818 dans l' interieur des États-Unis d' Amérique. Jour. Phys. 89: 96-107.

Rydberg, P. A. 1909. Distichlis dentata sp. nov. Bull. Torrey Bot. Club 36: 536.

Rzedowski, J. 2001. Distichlis. In: Rzedowski, G. C. de, J. Rzedowski y colaboradores. Flora Fanerogámica del Valle de México. 2a ed. Instituto de Ecología, A.C. y Comisión Nacional para el Conocimiento y Uso de la Biodiversidad. Pátzcuaro, Michoacán, México. 1406 pp. 
Villamil, C. B. 1969. El género Monanthochloë (Gramineae). Estudios morfológicos y taxonómicos con especial referencia a la especie argentina. Kurtziana 5: 369-391.

Watson, L. y M. J. Dallwitz. 1992. The grass genera of the World. C.A.B. International. Oxford. 1038 pp.

Zuloaga, F. O., E. G. Nicora, Z. E. Rúgolo de Agrasar, O. Morrone, J. Pensiero y A. M. Cialdella. 1994. Catálogo de la familia Poaceae en la República Argentina. Monogr. Syst. Bot. Missouri Bot. Gard. 47: 1-178. 

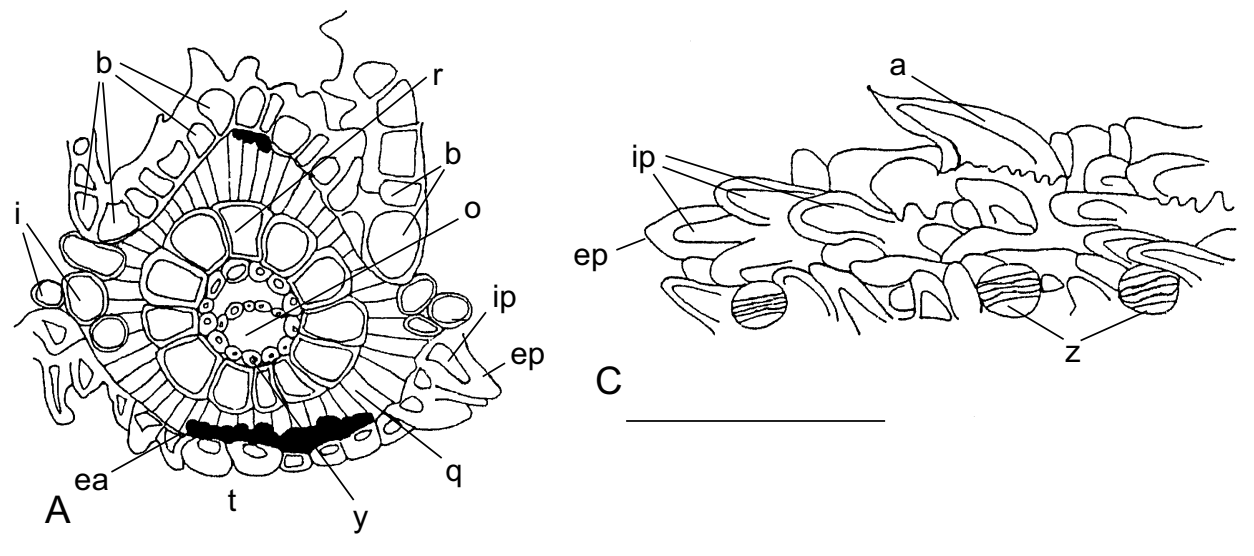

C
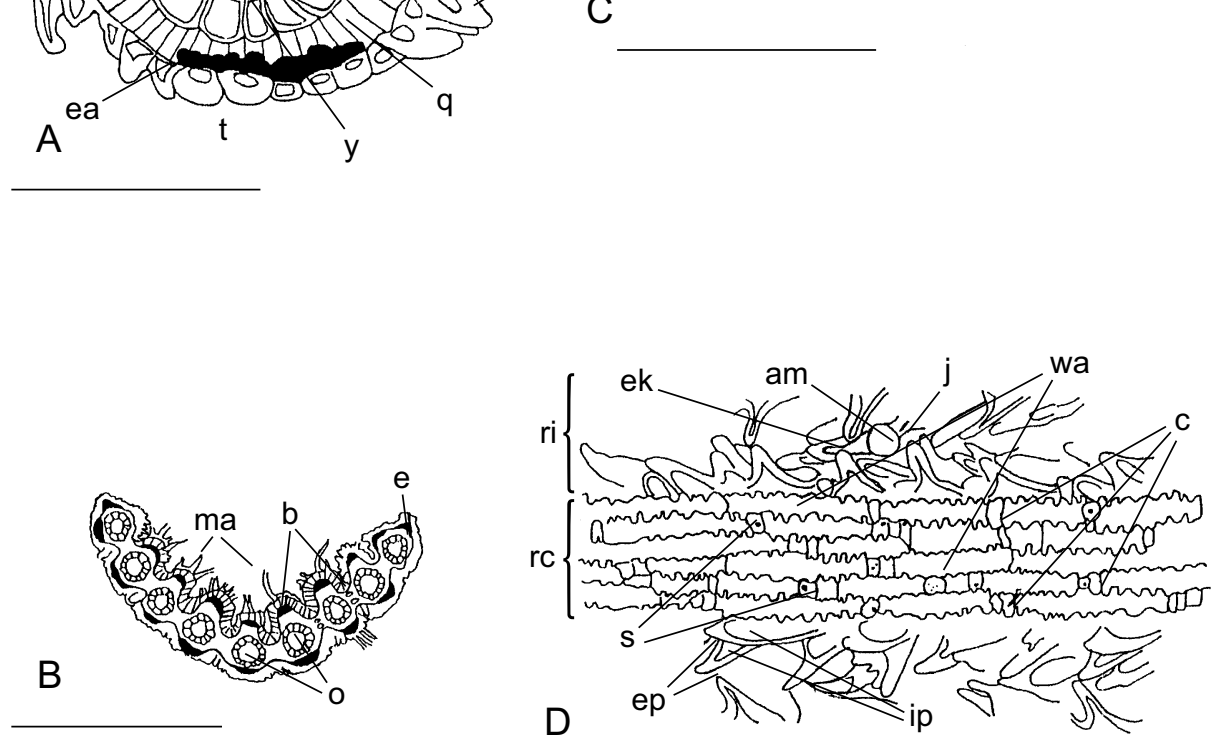

Fig. 1. Anatomía de la hoja de Distichlis australis. A. vena media; B. corte transversal; C. margen de la epidermis abaxial; D. epidermis abaxial. a = aguijón marginal, am = célula apical del micropelo, $\mathrm{b}=$ células buliformes, $\mathrm{c}=$ células de corcho, $\mathrm{e}=$ esclerénquima marginal, ea = esclerénquima abaxial de la costilla de la vena media, ek = exterior de la papila asociada a los micropelos, ep = exterior de las papilas, $\mathrm{i}=$ células incoloras, $\mathrm{ip}=$ interior de las papilas, $\mathrm{j}$ $=$ cuello sobresaliente de la célula basal del micropelo, $\mathrm{ma}=$ macropelos adaxiales, $\mathrm{o}=$ haces vasculares secundarios, $\mathrm{q}=$ vaina de clorénquima, $\mathrm{r}=$ vaina externa, $\mathrm{rc}=$ región costal, $\mathrm{ri}$ = región intercostal, $\mathrm{s}=$ cuerpos de sílice, $\mathrm{t}=$ epidermis abaxial de la costilla central, $\mathrm{wa}=$ células largas costales, $\mathrm{y}=$ vaina interna, $\mathrm{z}=$ células subsidiarias del complejo estomático. $A$. A. Beetle 495 UC, CAS-DS ${ }^{\lambda}$. Escala: A, C y D $=0.1 \mathrm{~mm}, \mathrm{~B}=0.4 \mathrm{~mm}$. 

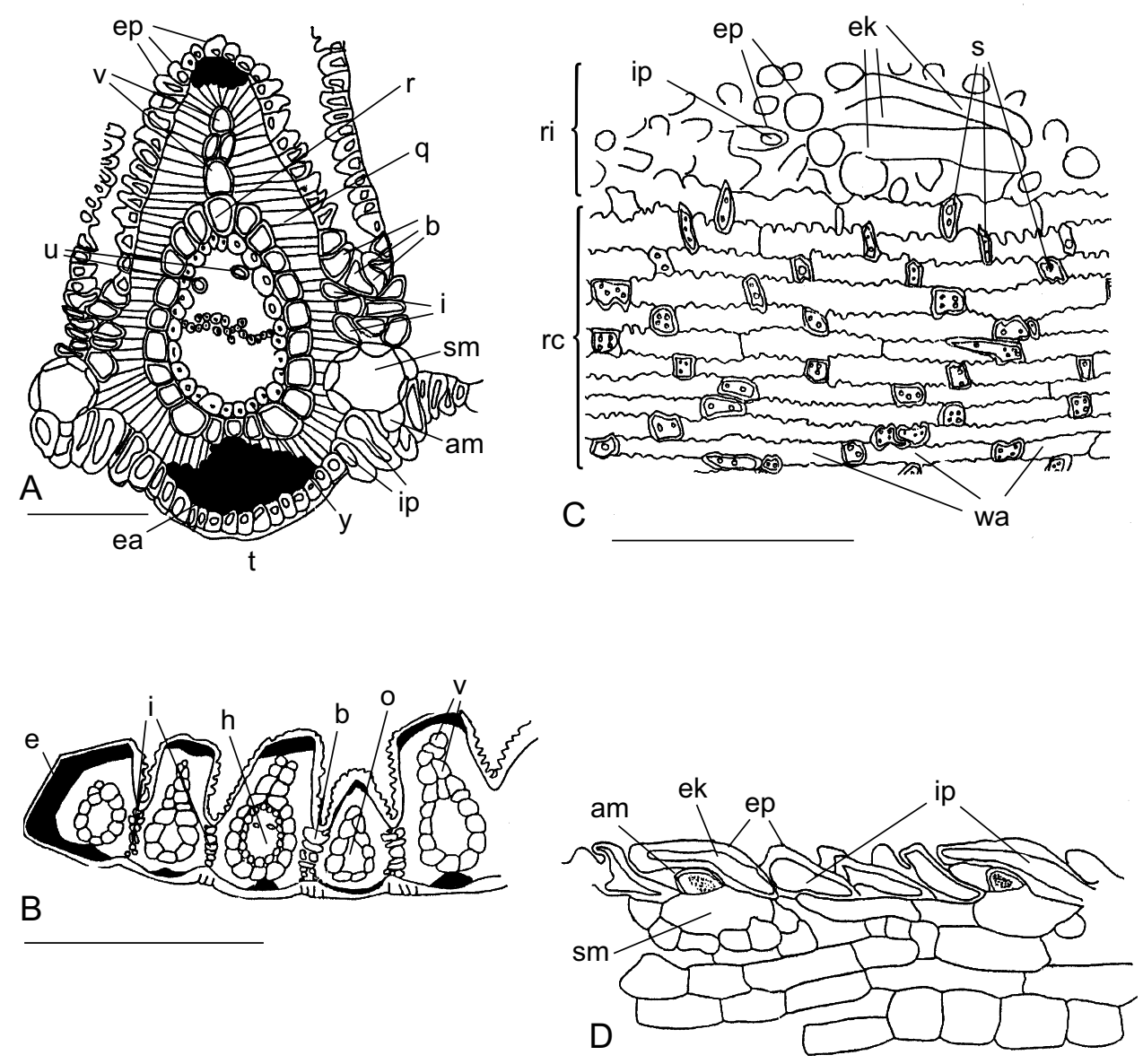

Fig. 2. Anatomía de la hoja de Distichlis palmeri. A. vena media; B. corte transversal; C. epidermis abaxial; D. corte longitudinal. am = célula apical del micropelo, $b=$ células buliformes, e = esclerénquima marginal fusionado al esclerénquima adaxial y abaxial de la misma costilla marginal, ea = esclerénquima abaxial de la costilla de la vena media, ek $=$ exterior del grupo de las papilas asociadas al micropelo cubriendo completamente esta estructura, ep $=$ exterior de las papilas, $\mathrm{h}=$ haz vascular primario, $\mathrm{i}=$ células incoloras, ip $=$ interior de las papilas, $\mathrm{o}=$ haz vascular secundario, $\mathrm{q}=$ vaina de clorénquima, $\mathrm{r}=$ vaina externa, $\mathrm{rc}=$ región costal, $\mathrm{ri}=$ región intercostal, $\mathrm{s}=$ cuerpos de sílice, $\mathrm{sm}=$ célula basal del micropelo, $\mathrm{t}=$ epidermis abaxial de la costilla central, $\mathrm{u}=$ vasos de metaxilema, $\mathrm{v}=$ extensión de la vaina externa, wa $=$ células largas costales, $\mathrm{y}=$ vaina interna. M. Equihua et al. $s / n$ MEXU $\widehat{\jmath}$. Escala: A, C y D $=0.1 \mathrm{~mm}, \mathrm{~B}=0.4 \mathrm{~mm}$. 

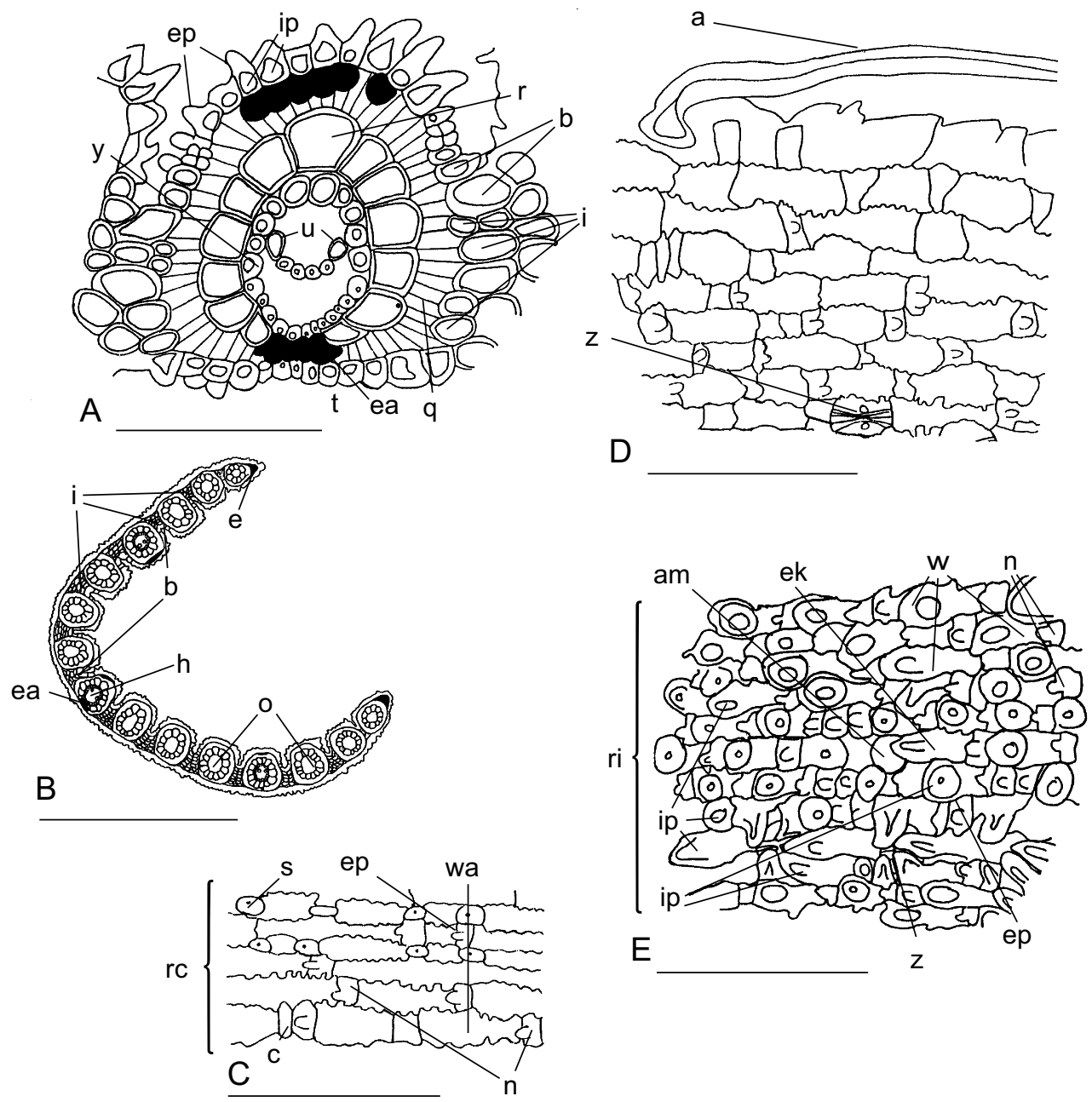

Fig. 3. Anatomía de la hoja de Distichlis humilis. A. vena media; B. corte transversal; C. región costal de la epidermis abaxial; D. margen de la epidermis abaxial; E. región intercostal de la epidermis abaxial. a = aguijón marginal, am = célula apical del micropelo, $b=$ células buliformes, $\mathrm{c}=$ célula de corcho, $\mathrm{e}=$ esclerénquima marginal, ea = esclerénquima abaxial de la costilla de la vena media, ek = exterior de la papila asociada al micropelo, ep = exterior de las papilas, $\mathrm{h}=$ haz vascular primario, $\mathrm{i}=$ células incoloras, $\mathrm{ip}=$ interior de las papilas, $\mathrm{n}$ $=$ células cortas normales, $\mathrm{o}=$ haces vasculares secundarios, $\mathrm{q}=$ vaina de clorénquima, $\mathrm{r}=$ vaina externa, $\mathrm{rc}=$ región costal, $\mathrm{ri}=$ región intercostal, $\mathrm{s}=$ cuerpo de sílice, $\mathrm{t}=$ epidermis abaxial de la costilla central, $\mathrm{u}=$ vasos de metaxilema, $\mathrm{w}=$ células largas intercostales, wa = célula larga costal, $\mathrm{y}=$ vaina interna, $\mathrm{z}=$ célula subsidiaria del complejo estomático. $S$. $G$. Beck 11777 US . . Escala: A, C, D y E $=0.1 \mathrm{~mm}, \mathrm{~B}=0.4 \mathrm{~mm}$. 

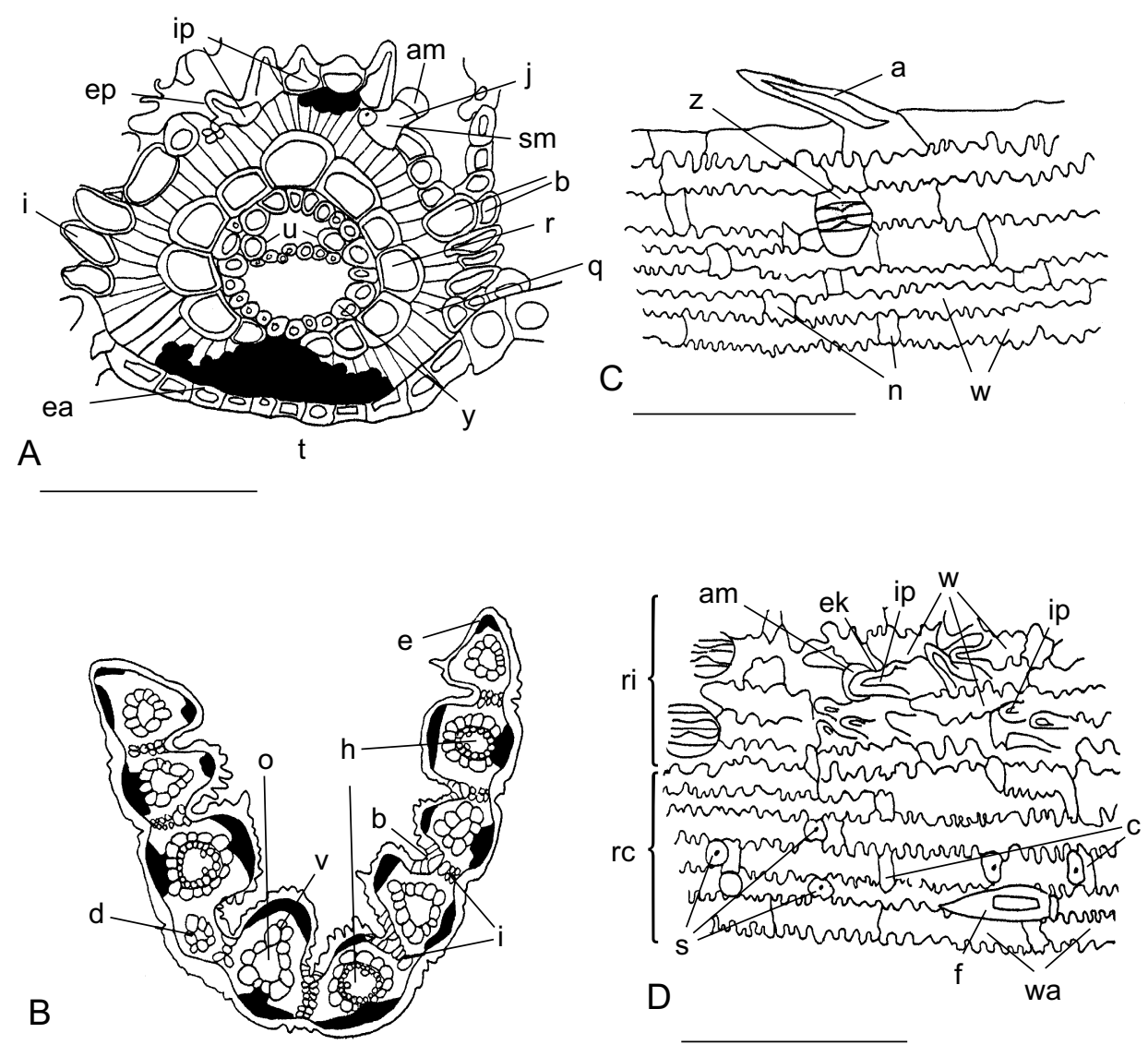

Fig. 4. Anatomía de la hoja de Distichlis scoparia var. erinacea. A. vena media; B. corte transversal; C. margen foliar; D. epidermis abaxial. a = aguijón marginal, am = célula apical del micropelo, $\mathrm{b}=$ células buliformes, $\mathrm{c}=$ células de corcho, $\mathrm{d}=$ haz vascular terciario, $\mathrm{e}=$ esclerénquima marginal, ea = esclerénquima abaxial de la costilla central, ek = exterior de la papila asociada al micropelo, ep = exterior de las papilas, $\mathrm{f}=$ aguijón epidérmico, $\mathrm{h}=$ haces vasculares primarios, $\mathrm{i}=$ células incoloras, $\mathrm{ip}=$ interior de las papilas, $\mathrm{j}=$ cuello sobresaliente de la célula basal del micropelo, $\mathrm{n}=$ células cortas normales, $\mathrm{o}=$ haz vascular secundario, $\mathrm{q}$ = vaina de clorénquima, $\mathrm{r}=$ vaina externa, $\mathrm{rc}=$ región costal, $\mathrm{ri}=$ región intercostal, $\mathrm{s}=$ cuerpos de sílice, $\mathrm{sm}=$ célula basal del micropelo, $\mathrm{t}=$ epidermis abaxial de la costilla central, $\mathrm{u}=$ vasos de metaxilema, $\mathrm{v}=$ extensión de la vaina externa, $\mathrm{w}=$ células largas intercostales, wa $=$ células largas costales, $\mathrm{y}=$ vaina interna, $\mathrm{z}=$ célula subsidiaria del complejo estomático H. A. Senn 4329 US . . Escala: A, C y D $=0.1 \mathrm{~mm}, \mathrm{~B}=0.4 \mathrm{~mm}$. 

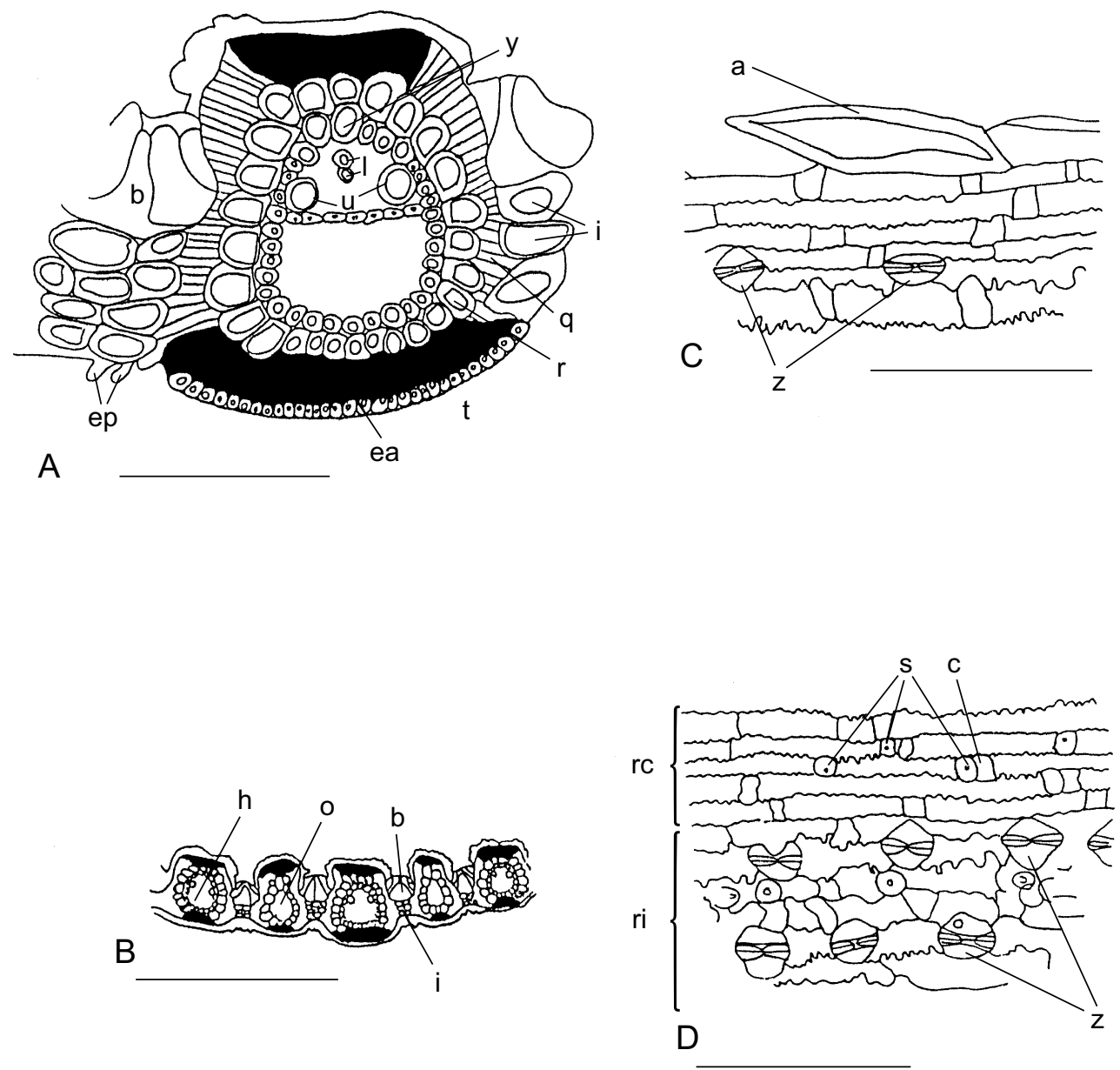

Fig. 5. Anatomía de la hoja de Distichlis spicata. A. vena media; B. corte transversal; C. margen de la lámina foliar; $\mathrm{D}$. epidermis abaxial. $\mathrm{a}=$ aguijón marginal, $\mathrm{b}=$ células buliformes, $\mathrm{c}=$ célula de corcho, ea = esclerénquima abaxial de la costilla central, ep = exterior de las papilas, $\mathrm{h}=$ haz vascular primario, $\mathrm{i}=$ células incoloras, $\mathrm{l}=$ laguna de protoxilema, $\mathrm{o}=\mathrm{haz}$ vascular secundario, $\mathrm{q}=$ vaina de clorénquima, $\mathrm{r}=$ vaina externa, $\mathrm{rc}=$ región costal, $\mathrm{ri}=$ región intercostal, $\mathrm{s}=$ cuerpos de sílice, $\mathrm{t}=$ epidermis abaxial de la costilla central, $\mathrm{u}=$ vasos de metaxilema, $\mathrm{y}=$ vaina interna, $\mathrm{z}=$ células subsidiarias del complejo estomático. $B$. J. Cox 2327 CHAPA of. Escala: A, C y D $=0.1 \mathrm{~mm}, \mathrm{~B}=0.4 \mathrm{~mm}$. 

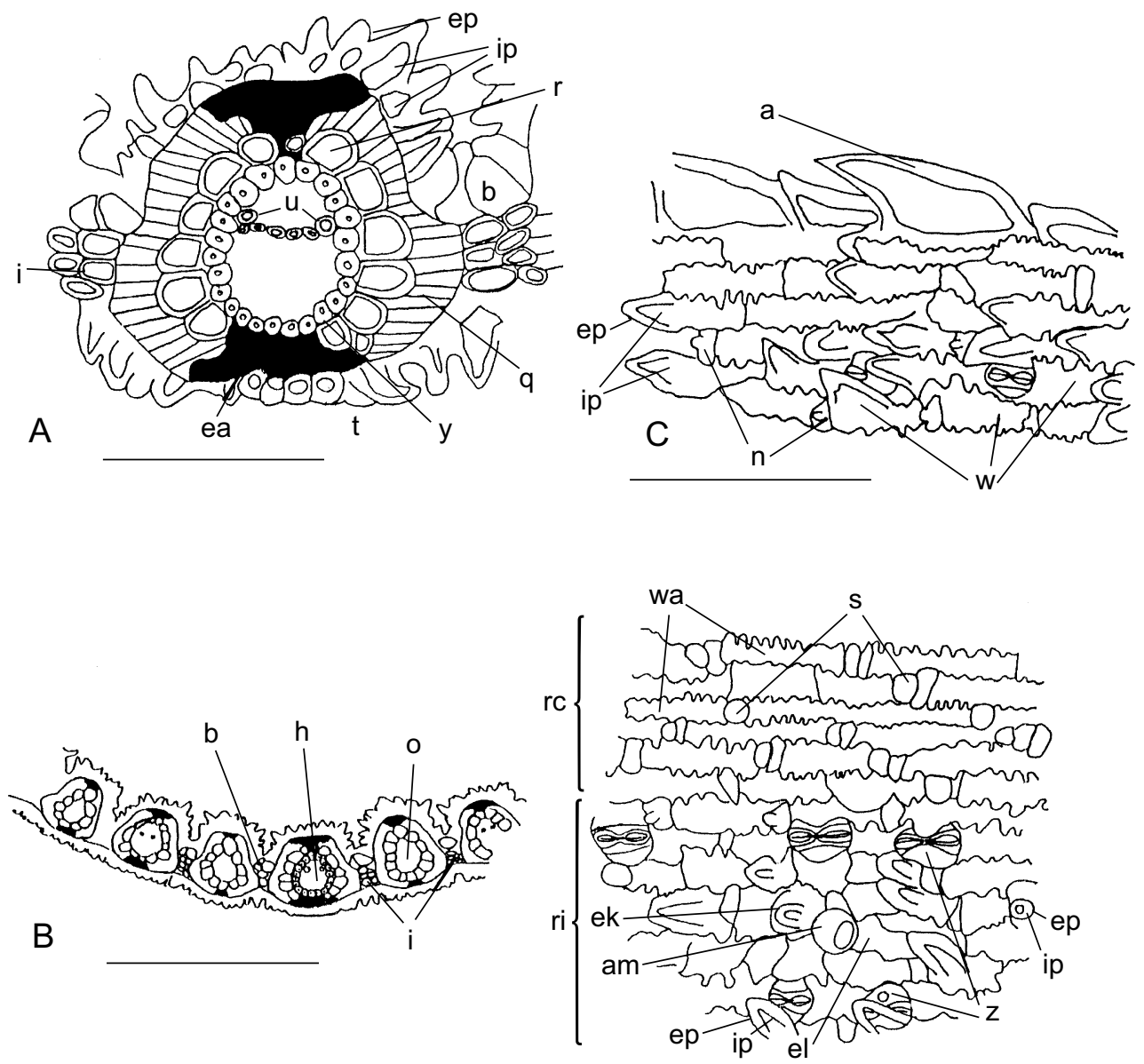

D

Fig. 6. Anatomía de la hoja de Distichlis spicata. A. vena media; B. corte transversal; C. margen de la lámina foliar; D. epidermis abaxial. a = aguijón marginal, am = célula apical del micropelo, $\mathrm{b}=$ células buliformes, ea = esclerénquima abaxial de la costilla central, ek = exterior de la papila asociada al micropelo, el = célula larga no papilosa asociada al micropelo, ep $=$ exterior de las papilas, $\mathrm{h}=$ haz vascular primario, $\mathrm{i}=$ células incoloras, $\mathrm{ip}=$ interior de las papilas, $\mathrm{n}=$ células cortas normales, $\mathrm{o}=$ haz vascular secundario, $\mathrm{q}=$ vaina de clorénquima, $\mathrm{r}=$ vaina externa, $\mathrm{rc}=$ región costal, $\mathrm{ri}=$ región intercostal, $\mathrm{s}=$ cuerpos de sílice, $\mathrm{t}=$ células abaxiales de las costilla central, $\mathrm{u}=$ vasos de metaxilema, $\mathrm{w}=$ células largas intercostales, $\mathrm{wa}$ = células largas costales, $\mathrm{y}=$ vaina interna, $\mathrm{z}=$ células subsidiarias del complejo estomático.

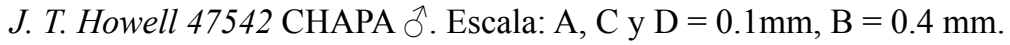



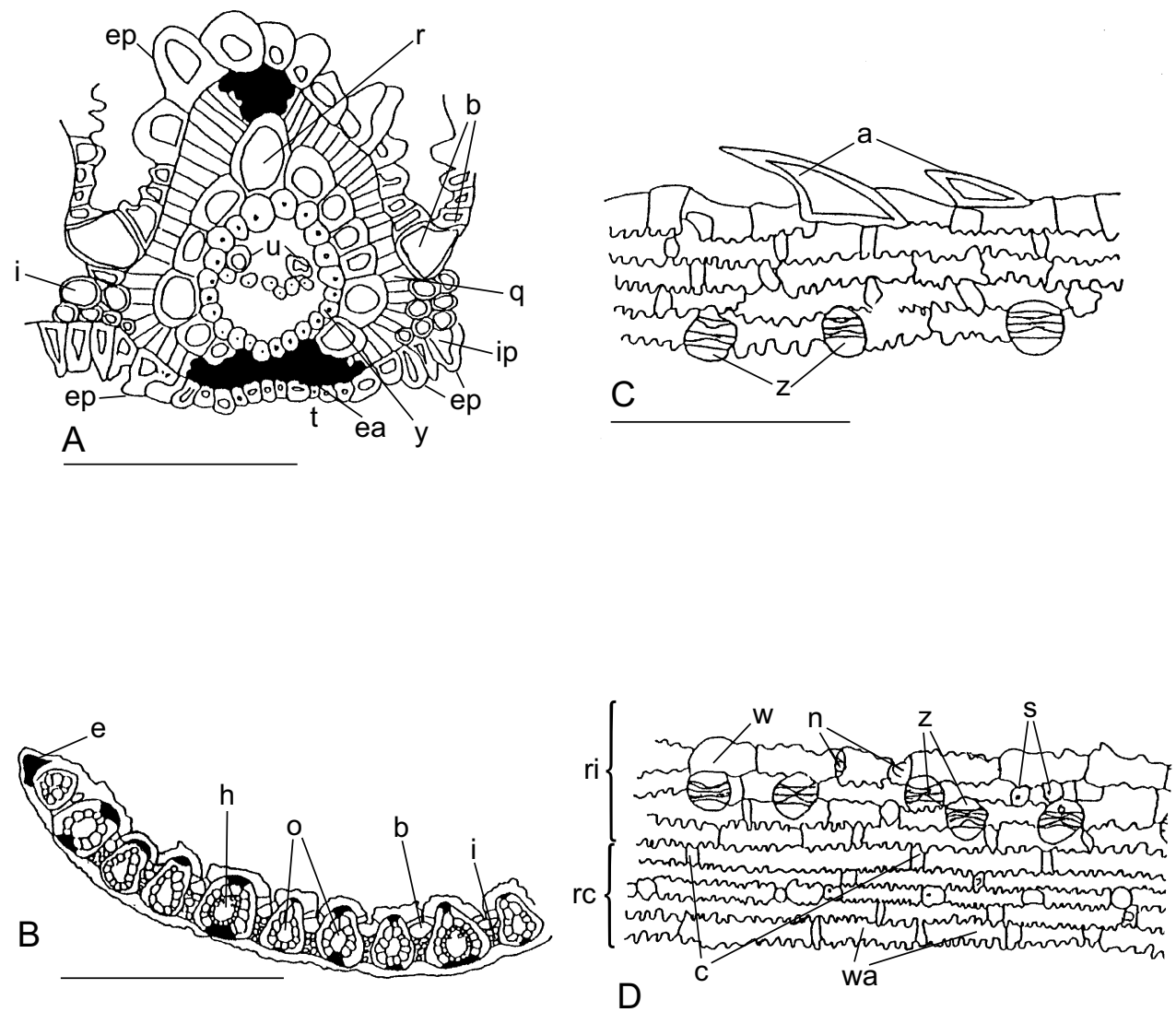

Fig. 7. Anatomía de la hoja de Distichlis spicata. A. vena media; B. corte transversal; C. región marginal de la epidermis abaxial; D. epidermis abaxial. a = aguijones marginales, $\mathrm{b}=$ células buliformes, $\mathrm{c}=$ células de corcho, $\mathrm{e}=$ esclerénquima marginal, ea $=$ esclerénquima abaxial de la costilla central, ep = exterior de las papilas, $\mathrm{h}=$ haz vascular primario, $\mathrm{i}=$ células incoloras, ip = interior de la papila, $\mathrm{n}=$ células cortas normales, $\mathrm{o}=$ haces vasculares secundarios, $\mathrm{q}=$ vaina de clorénquima, $\mathrm{r}=$ vaina externa, $\mathrm{rc}=$ región costal, $\mathrm{ri}=$ región intercostal, $\mathrm{s}=$ cuerpos de sílice, $\mathrm{t}=$ epidermis abaxial de la costilla central, $\mathrm{w}=$ célula larga intercostal, $\mathrm{wa}=$ células largas costales $\mathrm{y}=$ vaina interna, $\mathrm{z}=$ células subsidiarias del complejo estomático. $M$. LópezSoto 450 CHAPA $\delta$. Escala: A, C y D $=0.1 \mathrm{~mm}, \mathrm{~B}=0.4 \mathrm{~mm}$. 

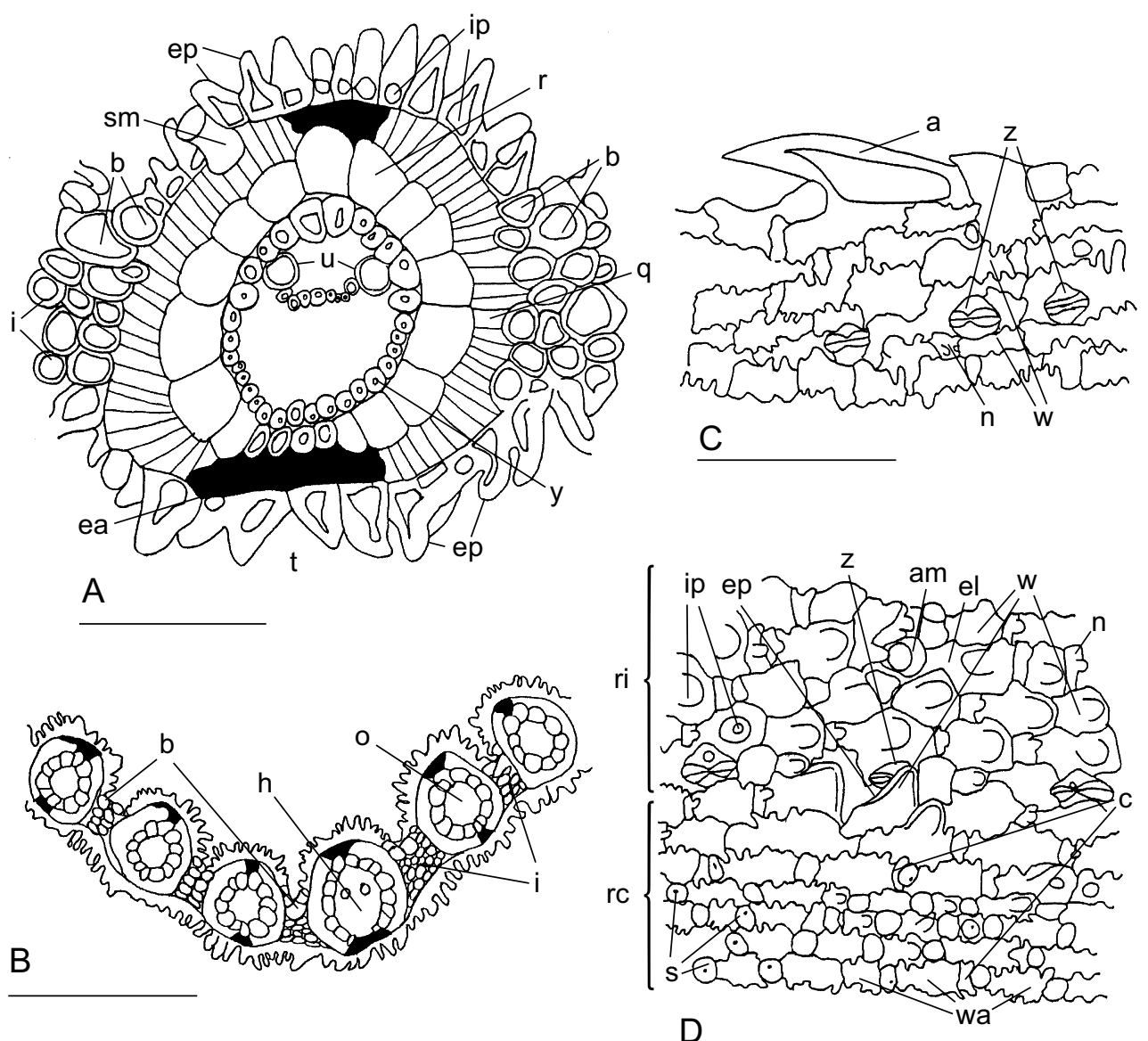

Fig. 8. Anatomía de la hoja de Distichlis spicata. A. vena media; B. corte transversal; C. región marginal de la epidermis abaxial; $\mathrm{D}$. epidermis abaxial. $\mathrm{a}=$ aguijón marginal, $\mathrm{am}=$ célula apical del micropelo, $\mathrm{b}=$ células buliformes, $\mathrm{c}=$ células de corcho, ea = esclerénquima abaxial de la costilla central, el = célula larga asociada al micropelo, ep = exterior de las papilas, $\mathrm{h}=$ haz vascular primario, $\mathrm{i}=$ células incoloras, $\mathrm{ip}=$ interior de las papilas, $\mathrm{n}=$ células cortas normales, $\mathrm{o}=$ haz vascular secundario, $\mathrm{q}=$ vaina de clorénquima, $\mathrm{r}=$ vaina externa, $\mathrm{rc}=$ región costal, $\mathrm{ri}=$ región intercostal, $\mathrm{s}=$ cuerpos de sílice, $\mathrm{sm}=$ célula basal del micropelo, $\mathrm{t}$ ) = epidermis abaxial de la costilla central, $\mathrm{u}=$ vasos de metaxilema, $\mathrm{w}=$ células largas intercostales, $\mathrm{wa}=$ células largas costales, $\mathrm{y}=$ vaina interna, $\mathrm{z}=$ células subsidiarias del complejo estomático. E. Werderman $431 \mathrm{UC} \widehat{\partial}$. Escala: A, C y D =0.1 mm, B = $0.4 \mathrm{~mm}$. 


\section{APÉNDICE}

Lista de especímenes utilizados en las observaciones anatómicas de la lámina foliar (abreviaturas según Holmgren et al., 1990)

Distichlis australis (Speg.) Villamil

Argentina: Provincia Chubut: A. A. Beetle 495 (CAS-DS ${ }_{0}$, UC).

Distichlis palmeri (Vasey) Fassett ex I.M. Johnst.

MÉxico: Estado de Baja California: Municipio Mulegé: I. L. Wiggins y D. B. Wiggins 18240 (MEXU đ)); I. M. Johnston 3491 (CAS-DS +); Municipio Ensenada: F. W.Gould y R. Moran 11928 (CAS-DS ${ }^{\Uparrow}$ ); R. Moran 4101 (DS ?); R. Moran 8646 (MEXU + ); R. Moran 12945 (CAS-DS +). Estado de Sonora: Municipio Guaymas: J. López-Portillo y B. Sánchez $s / n$ (MEXU) (sin inflorescencia); Municipio Puerto Peñasco: M. Equihua et al. $s / n$ (MEXU

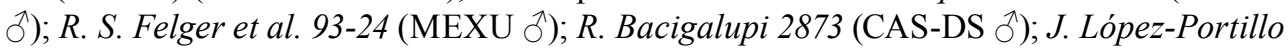
y B. Sánchez $s / n$ (MEXU) (sin inflorescencia); Municipio Hermosillo: R. S. Felger, M. Drees y N. Nicholson 74-32 (MEXU ㅇ); W. López F. 678 (MEXU); R. S. Felger et al. s/n (MEXU); Municipio San Luis Río Colorado: R. S. Felger y K. Cliffton 92-191 (MEXU ठొ).

Distichlis humilis Phil.

Bolivia: Provincia Ingavi: Departamento La Paz: S. G. Beck 11289 (US) (sin inflorescencia); Provincia L. Cabrera: Departamento Oruro: S. G. Beck 11777 (US + ).

Perú: Departamento Puno: R. S. Shepard 128 (US ${ }^{\circledR}$ ).

Distichlis scoparia (Nees ex Kunth) Arechav.

Argentina: Provincia La Pampa: Departamento Catrilo: J. Fortuna 15 (UC +). Provincia Chubut: Departamento Comodoro Rivadavia A. A. Beetle 138 (CAS-DS 9 ). Provincia Córdoba: Departamento Río Segundo: T. Stuckert 456 (UC ठ). Provincia Santa Cruz: A. Donat 82 (UC +); A. A. Beetle y D. Bignoli 112 (UC +). Provincia Neuquén: H. A. Senn 4329 (US 우).

URUGUAY: Departamento Canelones: H. Rosengurt B1886 (US ㅇ).

Distichlis spicata (L.) Greene

CAnAdÁ: Provincia British Columbia: J. A. Calder 9651 (UC + +); sin colector (UC ). Provincia Saskatchewan: I. W. Clokey 1622 (UC ठૈ).

Estados Unidos: Estado de Washington: $W$. J. Eyerdam $s / n$ (UC +). Estado de Nebraska: Lancaster Co: F. Cox y Dunn 1325 (CHAPA ठ̂); B. J. Cox 2327 (CHAPA ठ)). Estado de Maryland: Queen Anne Co: E. E. Terrell 3323 (CHAPA 9 ). Estado de California:

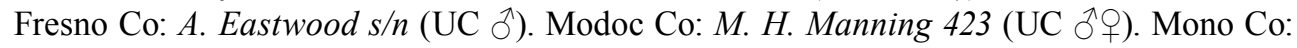
A. D. Gifford 879 (UC ㅇ); A. D. Gifford 880 (UC ?). San Bernardino Co: P. A. Munz 12,714 (UC ㅇ); S. B. Parish 11726 (UC ठ); J. B. Davy 2848 (UC ㅇ). Riverside Co: H. M. Hall

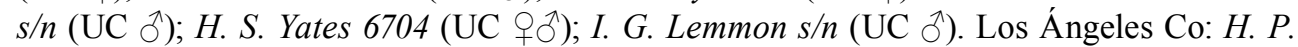

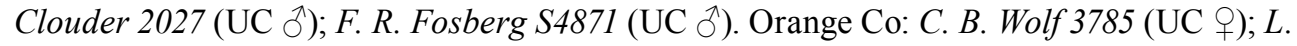


M. Booth 1116 (UC O). Mendocino Co: J. B. Davy y W. C. Blasdale 6125 (UC §̊). Humboldt Co: J. P. Tracy 6286 (UC )); sin colector (UC q); H. S. Yates 5859 (UC q); sin colector (UC §̊); J. B. Davy y W. C. Blasdale 6211 (UC + $)$. San Mateo Co: J. B. Davy s/n (UC + ). Monterey Co: C. A. Graham s/n (UC đ̃); J. B. Davy $s / n$ (UC +); E. Lee y H. Mason 9040

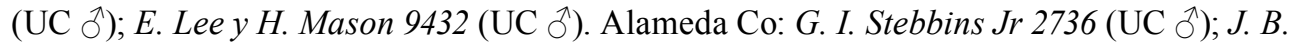
Davy 856 (UC +). Marin Co: L. Koch 1006 (UC ㅇ); J. B. Davy 4312 (UC ㅇ). San Diego Co: C. V. Meyer 454 (UC ㅇ); S. G. Stokes s/n (D-S 워); I. L. Wiggins 3015 (UC 우); C. V. Meyer 454 (UC q). Stanislaus Co: H. K. Sharsmith 1750 (UC) (sin inflorescencia); H. K. Sharsmith 3777 (UC 우). Kings Co: A. A. Beetle 2964 (UC 위). Douglas Co: W. D. Thomas 72 (UC ㅇ).

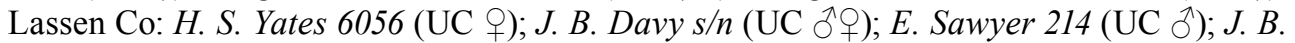

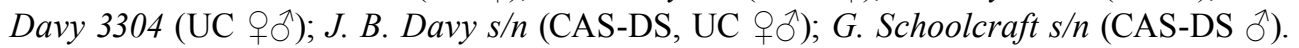
Placer Co: B. Crampton 3721 (CAS-DS +). Kern Co: J. B. Davy 2446 (UC đ̊); J. B. Davy

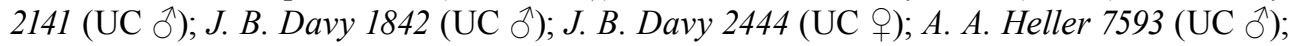
J. T. Howell 47542 (CHAPA §). Ventura Co: A. Simontacchi 112 (UC §̊); Sanford $s / n$ (UC +). Estado de Utah: M. E. Jones 5643 (UC §). Estado de Colorado: Moffat Co: C. L. Porter

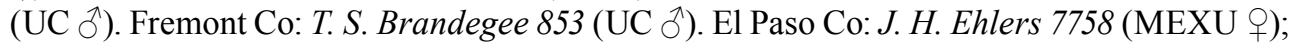

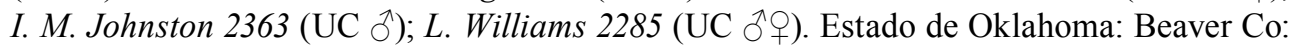
M. Huft y J. Estes 1387 (CHAPA +). Estado de North Carolina: Brunswick Co: C. R. Bell $s / n$ (UNC ). Estado de South Carolina: Beaufort Co: J. R. Bozeman et al. 11515 (UNC ). Estado de Arizona: Coconino Co: J. R. Reeder y C. G. Reeder 1690 (CHAPA §̋). Estado de Texas: Sweetwater Co: A. Nelson 3722 (UC ). Pecos Co: B. C. Tharp $43 A 6$ (MEXU §)). J. A. Villarreal et al. $s / n$ (CHAPA §). Galveston Co: $S$. L. Hatch 5764 (CHAPA §)). Estado de Georgia: McIntosh Co: W. H. Duncan 20432 (UNC ठ̊).

MÉxıCO: Estado de Baja California: Municipio Ensenada: Wiggins y Gillespie 3920 (MEXU + ); A. A. Beetle M-2770 (CHAPA O); M. Montoya A. 32 (MEXU đ̃); I. L. Wiggins y D. B. Wiggins 15946 (MEXU ठ); R. Guzmán M. 2751 (MEXU đ); R. Moran 3037 (UC) (sin inflorescencia); Municipio Mexicali: D. J. Pinkava y C. Minckley 3634 (MEXU ふ̋); Wiggins y Gillespie 4171 (MEXU +); Municipio Mulegé: A. A. Beetle M-2393 (CHAPA ठ̊); A. Preciado 65 (MEXU )); V. Morales 184 (MEXU $\bigcirc$ ); A. W. Anthony 139 (CAS-DS; UC §ึ). Estado de Sonora: Municipio Ímuris: J. R. Reeder y R. S. Felger 8125 (MEXU đ̂); Municipio San Luis Río Colorado: R. S. Felger y T. Bowen 86-530 (MEXU đ̊). Estado de Chihuahua: Municipio Delicias: S. D. Koch 74153 (CHAPA §̂); M. Carrasco H. 4 (CHAPA đ̋). Estado de Coahuila: Municipio Cuatrociénegas: E. Hernández X. y V. Mathus L. N-2032 (CHAPA §). Estado de Durango: Municipio Nombre de Dios: S. González 1212 (CHAPA +); E. Palmer 385 (MEXU §̊); Miles y W. Johnson 1763 (MEXU ठ̊); S. González y S. Acevedo 2724 (CHAPA †). Estado de Nuevo León: Municipio Galeana: E. Hernández X. y V. Mathus L. N-2047 (CHAPA); M. Siqueiros 296 (MEXU ठ̊). Estado de Zacatecas: Municipio Ojo Caliente: J. J. Balleza C. 1989 (CHAPA ठ઼); Rzedowski 12058 (CHAPA ठ̋). Estado de San Luis Potosí: Municipio Santo Domingo: J. J. Macías 10 (MEXU đ̂); Municipio Matehuala: G. Vázquez 35 (MEXU §). Estado de Tamaulipas: Municipio Altamira: D. Baro 441 (CHAPA ); D. Baro 380 (MEXU +). Estado de Guanajuato: Municipio Acámbaro: R. Guzmán M. 4454 (MEXU §); Municipio Valle de Santiago: L. I. Aguilera G. 179 (CHAPA ( ). Estado de Jalisco: Municipio Villa Corona: L. M. V. de Puga 3156 (CHAPA +); Municipio Zacoalco de Torres: L. M. V. de Puga 2850 (CHAPA đ̋). Estado de Michoacán: Municipio Cuitzeo: A. M. Benítez 
223-AMB (MEXU ठ̊); Rzedowski 39647 (CHAPA ふ̋); Rzedowski 44191 (CHAPA †); A. A. Beetle M-7128 (MEXU §)); Municipio Copándaro: Rzedowski 40280 (CHAPA §̋). Estado de México: Municipio Texcoco: Rzedowski 16266 (CHAPA đ̃); Sánchez M. s/n (CHAPA †); M. Gómez-Sánchez y M. González L. 501 (CHAPA §̂); Contreras 55 (MEXU †); S. D. Koch 7412 (CHAPA + ); R. Rascón R. 42 (CHAPA + ); M. López-Soto 450 (CHAPA ठ̋); $M$. López-Soto 451 (CHAPA + ); M. López-Soto 452 (CHAPA q); M. López-Soto 453 (CHAPA ); Municipio Ecatepec de Morelos: Rzedowski 30455 (MEXU §̋); Municipio Tlalnepantla: J. R. Rose et al. 8393 (MEXU +); Municipio Toluca: A. S. Hitchcock 6921 (MEXU $_{\text {); }}$ ) Municipio La Paz: C. de La Mora 7 (MEXU §̂). Distrito Federal: Delegación Tláhuac: $M$. $S$. Romero COCODA 23 (MEXU ठ̊); C. G. Pringle 6640 (MEXU đ̊); R. Méndez I. 1 (MEXU §); C. G. Pringle 6640 (UC §ै). Estado de Puebla: Municipio Oriental: S. Aguirre y C. S-133 (MEXU ठ̂); F. G. Medrano et al. 9301 (MEXU đ̊); Municipio San José Chiapa: S. Aguirre S-23 (CHAPA §̂); J. Rzedowski 32928 (CHAPA Ø̋); Municipio Zinacátepec: M. L. Aragón et al. 1118 (MEXU §̂); Municipio Tepeyahualco: R. l. Guzmán 4965 (MEXU); A. GómezPompa 3847 (MEXU §ึ). Estado de Tlaxcala: Municipio El Carmen Aztama: G. Ramos E. 69 (MEXU +). Estado de Yucatán: Municipio Progreso: J. R. Swallen 2925 (MEXU đ̂); Municipio Telchac: M. A. P. Lara 196 (MEXU +); F. Rodríguez 151 (CHAPA). Estado de Quintana Roo: Municipio Vigía Grande: E. Cabrera 3422 (MEXU q). Norte de México: $C$. Wright 2034 (UC क); C. Wright 2043 (UC ठ̊).

Ecuador: Provincia Cotopaxi: G. W. Prescott 374 (CAS-DS). Provincia Imbambura: Y. Mexia 7372 (UC) (sin inflorescencia).

Perú: Provincia Trujillo: Departamento La Libertad: C. R. Wort 8890 (CAS-DS, UC †); E. Anderson $s / n$ (UC ㅇ).

Chile: Provincia Atacama: Departamento Copiapó: E. Werdermann 431 (UC ふै); E. Werderman 997 (UC ).

Bolivia: Departamento Cochabamba: J. Steinbach 8745 (UC ठึ).

Argentina: Provincia Buenos Aires: A. L. Cabrera 5812 (UC ঐ); W. J. Eyerdam 23284 (UC §ึ). Provincia Chubut: Departamento Viedma: W. J. Eyerdam et al. 23575 (UC §). Departamento Rawson: W. J. Eyerdam 23597 (UC ठ̊). Provincia Jujuy: Departamento Tilcara: Meyer 3137 (UC ô). Departamento Tumbaya: M. C. Lippman, s/n (UC) (sin inflorescencia). Departamento Isubaya: S. Venturi 4899 (UC +). Provincia Mendoza: Departamento Las Heras: F. A. Barkley s/n (UC §̂); Departamento Comodoro Rivadavia: A. A. Beetle 138 (CAS-DS + ); A. A. Beetle y D. Bignoli 162 (UC + ); A. A. Beetle 576 (CASDS; UC + ). Provincia Santa Cruz: A. A. Beetle y D. Bignoli 113 (UC ঐ); W. J. Eyerman et al. 24515 (UC §). Provincia Tucumán: Rodríguez 190 (US đ̊). Provincia Corrientes: Departamento Burucuyá: T. M. Pedersen 4423 (UC + ).

URuguay: Departamento Montevideo: S. José 580 (UC ふ̋). 
